\title{
Arming oHSV with ULBP3 drives abscopal immunity in lymphocyte-depleted glioblastoma
}

\author{
Hans-Georg Wirsching, ${ }^{1,2}$ Huajia Zhang, ${ }^{3}$ Frank Szulzewsky, ${ }^{1}$ Sonali Arora, ${ }^{1,4}$ Paola Grandi, ${ }^{5,6}$ \\ Patrick J. Cimino, ${ }^{1,7}$ Nduka Amankulor, ${ }^{6}$ Jean S. Campbell, ${ }^{3}$ Lisa McFerrin,, ${ }^{1,4}$ Siobhan S. Pattwell, ${ }^{1}$ \\ Chibawanye Ene, ${ }^{1,8}$ Alexandra Hicks, ${ }^{9}$ Michael Ball, ${ }^{9}$ James Yan, ${ }^{1}$ Jenny Zhang, ${ }^{1}$ Debrah Kumasaka, \\ Robert H. Pierce, ${ }^{3}$ Michael Weller, ${ }^{2}$ Mitchell Finer, ${ }^{9}$ Christophe Quéva, ${ }^{9}$ Joseph C. Glorioso, ${ }^{5}$ \\ A. McGarry Houghton, ${ }^{3}$ and Eric C. Holland ${ }^{1,4,8,10}$ \\ ${ }^{1}$ Human Biology Division, Fred Hutchinson Cancer Research Center, Seattle, Washington, USA. ${ }^{2}$ Department of Neurology \\ and Brain Tumor Center, University Hospital Zurich and University of Zurich, Zurich, Switzerland. ${ }^{3}$ Clinical Research \\ Division and ${ }^{4}$ Seattle Translational Tumor Research, Fred Hutchinson Cancer Research Center, Seattle, Washington, USA \\ ${ }^{5}$ Department of Microbiology and Molecular Genetics and 'Department of Neurosurgery, School of Medicine, University of \\ Pittsburgh, Pittsburgh, Pennsylvania, USA. 'Department of Pathology, Division of Neuropathology, and ${ }^{8}$ Department of \\ Neurosurgery, University of Washington, Seattle, Washington, USA. ${ }^{9}$ Oncorus, Cambridge, Massachusetts, USA. ${ }^{10}$ Alvord \\ Brain Tumor Center, University of Washington, Seattle, Washington, USA.
}

Oncolytic viruses induce local tumor destruction and inflammation. Whether virotherapy can also overcome immunosuppression in noninfected tumor areas is under debate. To address this question, we have explored immunologic effects of oncolytic herpes simplex viruses (oHSVs) in a genetically engineered mouse model of isocitrate dehydrogenase (IDH) wild-type glioblastoma, the most common and most malignant primary brain tumor in adults. Our model recapitulates the genomics, the diffuse infiltrative growth pattern, and the extensive macrophage-dominant immunosuppression of human glioblastoma. Infection with an oHSV that was armed with a UL16binding protein 3 (ULBP3) expression cassette inhibited distant tumor growth in the absence of viral spreading (abscopal effect) and yielded accumulation of activated macrophages and T cells. There was also abscopal synergism of oHSV ${ }^{\mathrm{LLBP3}}$ with anti-programmed cell death 1 (anti-PD-1) against distant, uninfected tumor areas; albeit consistent with clinical trials in patients with glioblastoma, monotherapy with anti-PD-1 was ineffective in our model. Arming oHSV with ULBP3 led to upregulation of antigen processing and presentation gene sets in myeloid cells. The cognate ULBP3 receptor NKG2D, however, is not present on myeloid cells, suggesting a noncanonical mechanism of action of ULBP3. Overall, the myeloid-dominant, anti-PD-1-sensitive abscopal effect of oHSVULBP3 warrants further investigation in patients with IDH wild-type glioblastoma.

Authorship note: HGW and $\mathrm{HZ}$ are co-first authors.

Conflict of interest: ECH has served as a consultant for Oncorus; ECH, JCG, and $\mathrm{PG}$ have received research support from Oncorus. JCG and PG are among the cofounders and scientific advisors for Oncorus. AH, MB, MF, and $\mathrm{CQ}$ are employed by Oncorus.

Copyright: (c) 2019, American Society for Clinical Investigation.

Submitted: February 18, 2019

Accepted: May 16, 2019

Published: June 11, 2019.

Reference information: /Cl Insight. 2019;4(13):e128217. https://doi. org/10.1172/jci.insight.128217.

\section{Introduction}

Glioblastoma, the most common primary malignant brain cancer in adults, is an invariably deadly disease with median overall survival in the range of 12 months (1). The advent of immunotherapy prompted a plethora of clinical trials seeking to overcome the distinctive immunosuppression of these tumors, but phase III immunotherapy clinical trials of various strategies in patients with glioblastoma have failed to prolong survival (2). The tumor microenvironment of glioblastoma is dominated by immunosuppressive tumor-associated microglia and macrophages (TAMs) that limit immune surveillance by lymphocytes (3).

Preclinical exploration of microenvironment-modulating therapies requires bona fide cancer models with histologic and immunologic features that resemble their human counterparts as closely as possible. Applying a sequential bioinformatics and genetic engineering approach to identify and subsequently model relevant oncogenic events, we have previously generated mouse glioblastoma models that develop in situ from each mouse's own cells and that are histologically and molecularly highly similar to human glioblastoma (4).

Promising response rates and translational data from early-phase biologic endpoint trials support the rationale and feasibility of virotherapy approaches to revert immunosuppression in glioblastoma (5-7). 
Oncolytic viruses are usually administered locally, either directly into the tumor or into the resection cavity during surgery. To be efficacious in patients with disseminated tumors or multifocal cancer, virotherapy will, however, need to induce distant anticancer immune responses beyond local tumor cell lysis.

Here, we explored localized and distant (abscopal) immunologic effects of oncolytic virotherapy in a genetically engineered, immunologically inert model of diffusely infiltrating glioblastoma.

\section{Results}

Immunologic properties of genetically engineered isocitrate dehydrogenase wild-type glioblastoma. The vast majority of glioblastomas are classified by the lack of isocitrate dehydrogenase (IDH) mutations and harbor gain of chromosome 7 , loss of heterozygosity of chromosome 10, and homozygous deletion of cyclin-dependent kinase inhibitor 2A (CDKN2A) (refs. 4, 8; Supplemental Figure 1A; supplemental material available online with this article; https://doi.org/10.1172/jci.insight.128217DS1). We have used the RCAS/tv-a gene transfer system in $N / t v-a C d k n 2 a^{-/-} P t e n^{f / f l} L u c^{L S L / L S L}$ (XFM-Luc) mice to generate IDH wild-type glioblastomas in situ from each mouse's own cells. Nestin-positive neural stem and progenitor cells in XFM-Luc mice were infected with RCAS-PDGF to model chromosome 7 gain and with RCAS-Cre to model loss of chromosome 10 (XFM-Luc:PDGF,Cre; ref. 4 and Supplemental Figure 1B).

Immunohistochemistry of mouse and human IDH wild-type glioblastomas suggested that key components of the immune cell compartment are similar between our genetically engineered mouse glioblastomas and their human counterparts. There was a vast abundance of ionized calcium-binding adaptor molecule 1-positive $\left(\mathrm{Iba}^{+}\right)$TAMs, which accumulated in zones of pseudopalisading necrosis, whereas $\mathrm{CD}^{+}$tumor-infiltrating lymphocytes were hardly detected (Figure 1A). Flow cytometry confirmed that XFM-Luc:PDGF,Cre glioblastomas had few if any lymphocytes and that the majority of CD45 $5^{+}$cells were of myeloid origin, predominantly comprising bone marrow-derived macrophages $\left(C D 45^{\mathrm{hi}} \mathrm{CD} 11 \mathrm{~b}^{+} \mathrm{Ly}-\right.$ $6 \mathrm{c}^{\mathrm{lo}} \mathrm{Ly} 6 \mathrm{~g}$, mean $53 \%$, Figure 1B). Consistent with the only completed phase III clinical trial of immune checkpoint inhibition in patients with glioblastoma (9), various immune checkpoint inhibitors had no effect on the survival of XFM-Luc glioblastoma-bearing mice (Figure 1C).

Our model reflects the immune phenotype of its human counterpart (3) and circumvents major limitations of commonly used syngeneic cancer models, including hypermutation, lymphocyte accumulation, and an inherent response to immune checkpoint inhibition $(10,11)$.

oHSVULBP3 leverages accumulation of activated TAMs. We have used this IDH wild-type glioblastoma model as a paradigm to develop an oncolytic herpes simplex virus (oHSV), which we optimized for the treatment of glioblastoma using a microRNA 124-based (miR-124-based) attenuation strategy (ref. 12, Supplemental Figure 2, A-E, and Supplemental Note 1). Moreover, as a means to augment an expected virotherapy-induced immune response, we generated an oHSV, which included a payload cassette to drive the expression of human UL16-binding protein 3 (ULBP3), a class 1 major histocompatibility complex-like (MHC-like) molecule and member of the family of ligands of the killer cell lectin-like receptor subfamily $\mathrm{K}$ member 1 (NKG2D). NKG2D-independent, proinflammatory effects on myeloid cells have been reported for ULBP3 but not for ULBP1 or ULBP2 (13), rendering ULBP3 our prime candidate payload to revert the macrophage-dominant immunosuppression of glioblastoma.

Mice bearing XFM-Luc:PDGF,Cre glioblastomas were randomized to undergo intratumor injection of PBS, oHSV, or oHSV ULBP3 (Figure 2A). oHSV ${ }^{\mathrm{ULBP} 3}$ prolonged median survival after treatment from 8 to 18 days (HR $0.28, P<0.001)$ and inhibited tumor growth, as assessed by luminescence imaging $(P=0.013)$, whereas oHSV lacking the ULBP3 payload cassette did not (HR 1.02, $P=0.94$; Figure 2B and Supplemental Figure 2F). Therefore, we focused our further efforts on $\mathrm{OHSV}^{\mathrm{ULBP} 3}$ to determine its utility as an investigational drug.

Histopathologic analysis of glioblastoma-bearing mice sacrificed on day 7 after intratumor injection of $\mathrm{oHSV}^{\mathrm{ULBP} 3}$ revealed necrotic areas in zones of viral replication in 4 of 4 analyzed mice, indicating oncolysis (Figure 2C). Viral replication and oncolysis were generally restricted to small foci surrounding the injection site. Immunohistochemistry staining identified accumulation of $\mathrm{Ibal}^{+}$myeloid cells in $\mathrm{EGFP}^{+}$zones of oHSV ${ }^{\mathrm{ULBP} 3}$ replication (Figure 2D). Computed imaging analyses of Iba1-stained tumor sections $(n=4$ per group, day 7 after viral infection) revealed that upon treatment with oHSVULBP3 the overall tumor area covered with $\mathrm{Iba}^{+}$cells increased by more than 2 -fold compared with PBS-injected tumors $(P=0.016)$, but oHSV lacking the ULBP3 expression cassette did not have that effect $(P=0.66$; Figure $2 \mathrm{E})$.

oHSV VLBP3 leverages accumulation of activated T cells. We considered adding ULBP3 primarily to strengthen oHSV-induced antiviral $\mathrm{T}$ cell responses because its cognate human receptor, NKG2D, amplifies the cyto- 
A
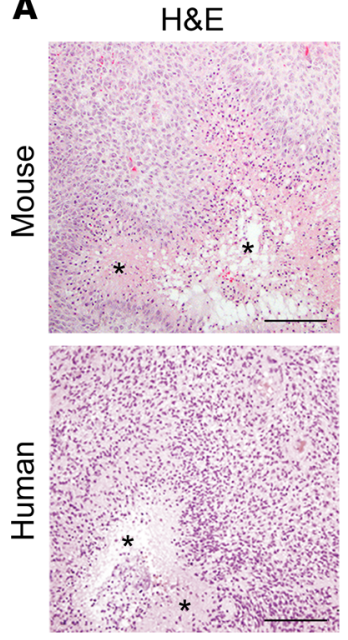

B

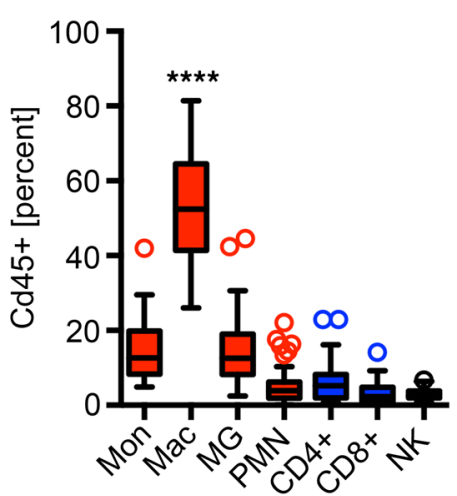

lba1
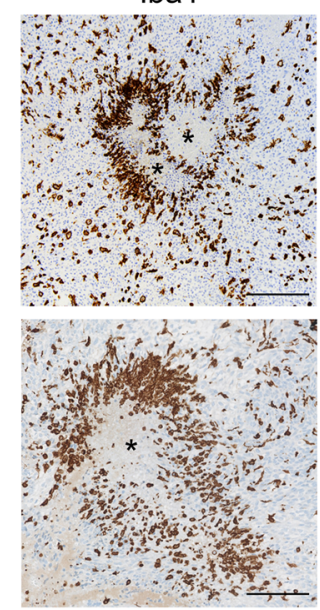

C

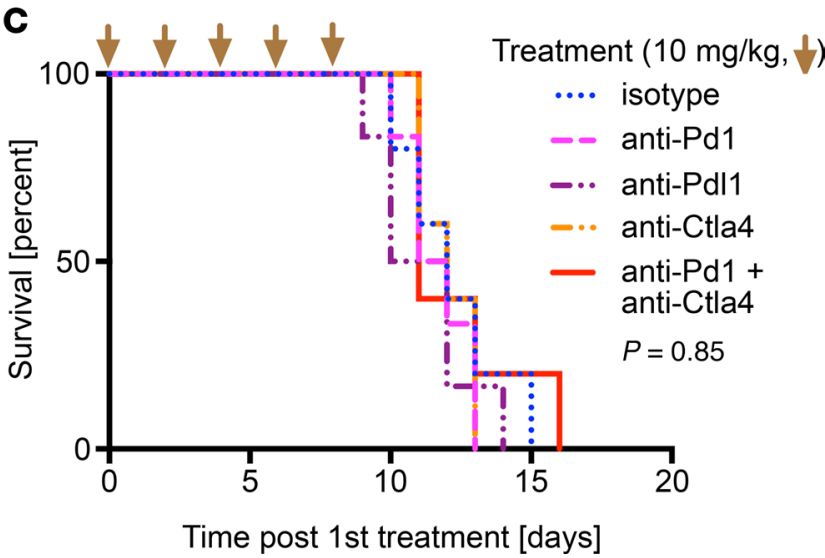

Figure 1. XFM-Luc:PDGF,Cre mouse glioblastomas immunologically resemble human glioblastoma. (A) Representative tumor sections of XFM-Luc:PDGF,Cre glioblastomas (mouse, $M, n=4$ ) and human IDH wild-type glioblastoma (human, $\mathrm{Hu}, n=4$ ). Staining by immunohistochemistry as indicated, with quantitation of (top) CD3 ${ }^{+}$cells in $5 \times 40$ high-power fields (HPFs) per sample and (bottom) the area covered by lba1+ TAMs $(n=4$ per group). Scale bar: $200 \mu \mathrm{m}$. 2-sided $t$ test. Asterisks indicate necrosis. (B) Flow cytometry of CD45 ${ }^{+}$cells in brain hemispheres bearing untreated XFM-Luc:PDGF,Cre glioblastomas. $n=46$ tumors from $n=8$ independent experiments. ${ }^{* * * *} P<$

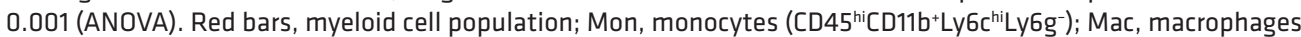

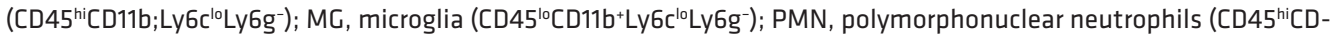

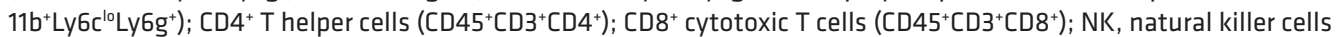
(CD45+CD49+). The box plots depict the minimum and maximum values (whiskers), the upper and lower quartiles, and the median. The length of the box represents the interquartile range. (c) Symptom-free survival of mice bearing XFM-Luc:PDGF,Cre glioblastomas treated with isotype control or indicated immune checkpoint inhibitory monoclonal antibodies (10 $\mathrm{mg} / \mathrm{kg}$ i.v. every other day, $n=5-6$ per group). Treatment began on day 14 after tumor initiation. Survival curves were compared using the log-rank test.

toxicity of activated $\mathrm{T}$ cells in addition to activating NK cells (14). There was indeed an increase in CD8 ${ }^{+}$ $\mathrm{T}$ cells in oHSV ${ }^{\mathrm{ULBP} 3}$-treated tumors (mean $\mathrm{CD}^{+}$cells per high-power field [HPF] in PBS vs. oHSV ${ }^{\mathrm{ULBP} 3}=$ 2 vs. $19 ; n=4$ per group; $P=0.015)$ but not in tumors treated with an oHSV that was lacking ULBP3 ( $P=$ 0.14; Figure $2 \mathrm{~F})$. The number of tumor-infiltrating FoxP3 ${ }^{+}$regulatory T cells increased from 1 to 7 per HPF upon treatment with oHSV ${ }^{\mathrm{ULBP} 3}(P=0.018)$, but the ratio of $\mathrm{CD}^{+}$to $\mathrm{FoxP}^{+}$cells increased from 2 to $3(P$ $=0.020$; Supplemental Figure $2 \mathrm{G})$. oHSV ${ }^{\mathrm{ULBP}}$-driven $\mathrm{CD}^{+} \mathrm{T}$ cell influx was further enhanced by cotreatment with anti-PD-1 $(P=0.003)$, but anti-PD-1 had no effect on $\mathrm{CD} 8^{+}$influx in the absence of virotherapy $(P=0.55$; Supplemental Figure $2 \mathrm{H})$. There was also synergism of $\mathrm{oHSV} \mathrm{ULBP}^{\mathrm{U}}$ and anti-PD-1 in promoting the $\mathrm{CD}^{+} \mathrm{CD} 44^{-} \mathrm{CD} 62 \mathrm{~L}^{-}$effector $\mathrm{T}$ cell phenotype from $1 \%$ in PBS-treated tumors to $15 \%$ of $\mathrm{CD}^{+}$cells in oHSV ${ }^{\mathrm{UBP} 3}$ plus anti-PD-1 cotreated tumors $(P=0.002)$. Interestingly, both treatments given as monotherapy had only minor effects on the effector T cell fraction (Supplemental Figure 2I). In contrast, flow cytometry determined there was only a minor increase in NK cells on day 7 after treatment with oHSV ${ }^{\text {ULBP3 }}$ 
A

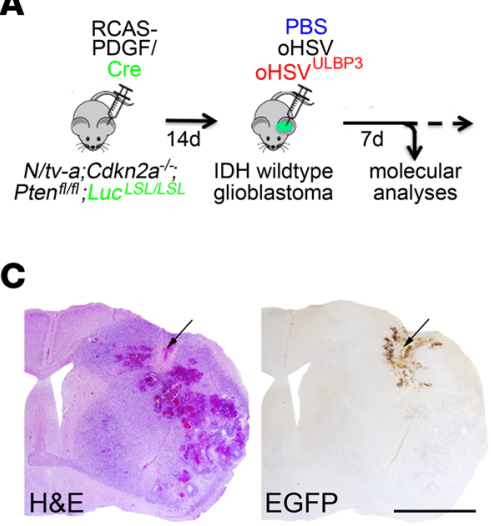

D

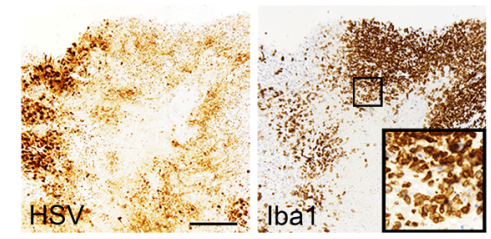

G

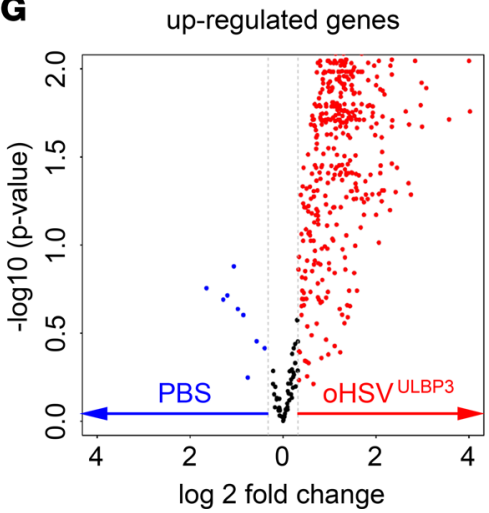

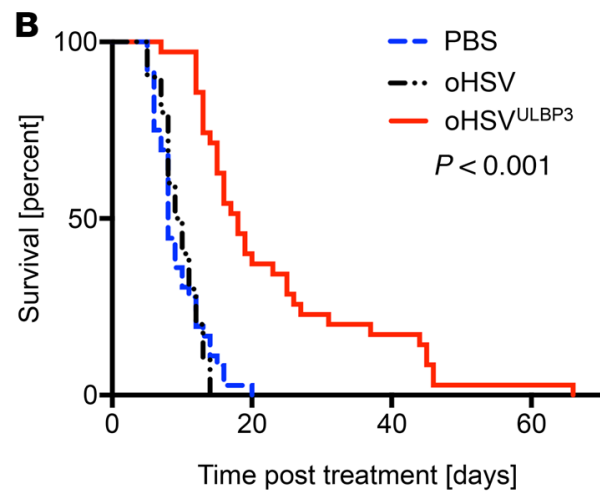

E
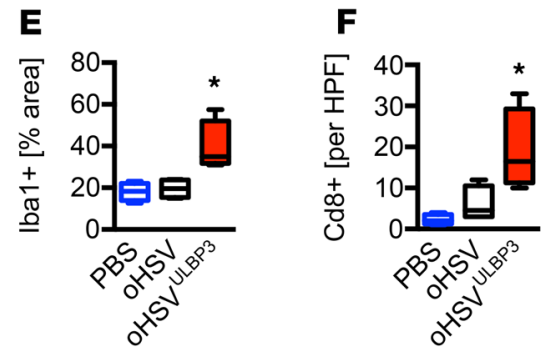

H

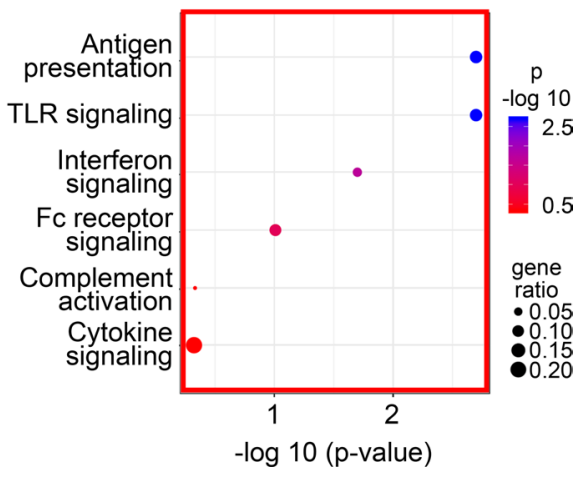

Figure 2. OHSV ${ }^{\mathrm{ULBP}}$ reverts immunologic inertness and prolongs survival of glioblastoma-bearing mice. (A) Experimental setup. (B) Symptom-free survival. PBS, $n=35$; oHSV, $n=10$; oHSVULBP3 $n=36$. Kaplan-Meier curves were compared using the log-rank test. (C) Representative H\&E staining and immunohistochemistry of EGFP in adjacent tissue slides of oHSVuLBP3-treated mouse glioblastomas. Arrows, localized hypocellular zone (left) colocalizing with EGFP+ $^{+}$viral replication (right). Scale bar: $2 \mathrm{~mm}$. (D) Representative immunohistochemistry stains of HSV antigens (left) and Iba1+ TAMs (right) in oHSVuLBP3-treated mouse glioblastomas. Inset, activated Iba1+ cells. Scale bar: $200 \mu \mathrm{m}$. (E and F) Tumor surface area covered by $\mathrm{Iba}^{+}$cells determined by automated image analysis $(\mathbf{E})$ and counts of the maximum number of $C D 8^{+} T$ cells detected per HPF $(\mathbf{F})$ in mouse glioblastomas 7 days after tumor injection with PBS or $1 \times 10^{6}$ PFU of indicated oHSVs. $n=4$ tumors per group; 2-tailed, unpaired $t$ test; ${ }^{*} P<0.05$. The box plots depict the minimum and maximum values (whiskers), the upper and lower quartiles, and the median. The length of the box represents the interquartile range. (C) Volcano plot of differentially expressed genes of the nCounter myeloid panel in PBS- versus

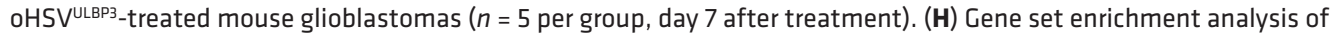
genes upregulated in oHSVuLpp3 (red in G).

$(P=0.026 ;$ Supplemental Figure 2J). This increase was accompanied by a decrease in CD69+ activated NK cells $\left(P=0.036\right.$; Supplemental Figure $2 \mathrm{~K}$ ) and loss of $\mathrm{Nkg} \mathrm{d}^{+}$cells (percentage of $\mathrm{Nkg}^{2} \mathrm{~d}^{+} \mathrm{NK}$ cells upon treatment with PBS vs. oHSV ${ }^{\mathrm{ULBP} 3}=21$ vs. $3 ; P<0.001$; Supplemental Figure $2 \mathrm{~L}$ ), indicating that NK cells were likely not the driving force of the observed survival benefit.

oHSVULBP3 promotes antigen presentation and TLR signaling. To characterize the effects of oHSVULBP3 on the tumor microenvironment in more detail, we performed nCounter gene expression analyses using 2 gene panels, myeloid innate immunity and immunology ( $n=5$ tumors per group and panel). 
Within the myeloid innate immunity gene panel ( $n=732$ genes), there were $n=461$ genes that were more than 2-fold upregulated and $n=10$ genes that were more than 2-fold downregulated in tumors injected with oHSV ${ }^{\mathrm{ULBP} 3}$ versus PBS (Figure $2 \mathrm{G}$ ). Gene set enrichment analysis of genes upregulated upon oHSV ${ }^{\mathrm{UBP} 3}$ treatment versus PBS identified the Gene Ontology (GO) terms antigen presentation $(P=0.002)$ and TLR signaling $(P=0.002 ;$ Figure $2 \mathrm{H})$. Both gene sets were also upregulated in tumors treated with oHSV ${ }^{\mathrm{ULBP} 3}$ versus oHSV (antigen presentation, $P=0.043$; TLR signaling, $P=0.043$ ), suggesting a ULBP3-mediated effect. Similar analyses of the immunology gene panel ( $n=545$ genes) in tumor-bearing mice treated with $\mathrm{OHSV}^{\mathrm{ULBP} 3}$ versus PBS identified enrichment of the Kyoto Encyclopedia of Genes and Genomes (KEGG) gene sets phagosome $(P=0.023)$ and confirmed enrichment of antigen processing and presentation $(P=0.004)$ as well as a trend for increased TLR signaling $(P=$ 0.065 ) upon oHSV ${ }^{\mathrm{ULBP} 3}$ treatment (Supplemental Figure $2 \mathrm{M}$ ).

The dominance of a myeloid immune response was surprising in the light of putative NKG2D-mediated effects of ULBP3. Because the cognate ULBP3 receptor, Nkg2d, is not present on myeloid cells, we assume there is a noncanonical mechanism of action of ULBP3. A previous study using the EL4 and RMA mouse tumor models demonstrated that ectopic expression of human ULBP3 in murine tumor cells results in tumor rejection but failed to demonstrate binding of ULBP3 to murine Nkg2d (13). Structure interaction modeling using the Protein Modeling Portal (15) predicted a weak, if any, physical interaction of human ULBP3 with human NKG2D and suggested that human ULBP3 did not bind to the mouse ortholog of NKG2D (Supplemental Figure 2N). Flow cytometry of human peripheral blood mononuclear cells and murine splenocytes using Fc-tagged recombinant ULBP3 con-

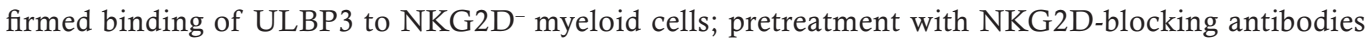
did not abrogate ULBP3 binding to myeloid cells (Supplemental Figure 2O). Collectively, these data suggest a noncanonical mechanism of action of ULBP3 that drives antigen processing and presentation through interaction with an unknown receptor on myeloid cells. This notion is supported by publically available data from mass spectrometry-based affinity capture experiments in human cell lines, which identified 19 ULBP3 binding partners (16) that are also enriched for the GO term antigen processing and presentation (Supplemental Figure 2P). In humans, ULBP3 is in addition a cognate ligand of NKG2D, suggesting that in patients, oHSV ${ }^{\mathrm{ULBP} 3}$ may exert additional favorable immunomodulating effects through activation of $\mathrm{NK}$ and $\mathrm{T}$ cells via NKG2D.

However, although our study supports a model of pleiotropic effects of ULBP3, antitumor activity could also result from the generation of opsonizing antibodies directed against soluble Nkg2d ligands (17). More in-depth research will be required to determine the precise mechanism by which ULBP3 activates macrophages.

Abscopal accumulation of activated T cells upon localized oHSVULPP infection. We reasoned that virotherapy could be effective only against metastasizing or diffusely infiltrating cancers such as glioblastoma if an anticancer immune response were elicited not only locally but also distant from the site of tumor infection, i.e., by exerting an abscopal effect. To model oHSV-infected and uninfected tumor portions, we generated 2 spatially segregated tumors in both hemispheres of XFM-Luc mice. In this modified bilateral glioblastoma model, luciferase was activated only in the untreated tumor portion (Supplemental Figure 3A), allowing us to monitor abscopal growth independent of local effects of virotherapy.

We then used this model to explore abscopal effects of localized oHSV ULBP3 therapy (Figure 3A). Unilateral treatment with oHSV ${ }^{\mathrm{ULBP} 3}$ inhibited contralateral tumor growth (Figure 3B) and prolonged survival (HR 0.49, $P=0.003$; Figure 3C). Of note, contralateral $\mathrm{Luc}^{+}$tumor lesions were survival limiting (ref. 4 and Supplemental Figure $3 \mathrm{~B}$ ), indicating that the survival benefit from unilateral treatment was due to abscopal inhibition of tumor growth. The lack of any survival effect of oHSV without the ULBP3 expression cassette (Figure 2) and the lack of viral replication in normal brain cells (Supplemental Note 1) suggests that infection and replication of $\mathrm{OHSV}^{\mathrm{ULBP} 3}$ in the tumor was a prerequisite for inducing an abscopal effect. There was also abscopal sensitization to immune checkpoint inhibition with anti-PD-1, which translated into prolonged survival among those mice that survived for at least 7 days (HR $0.31, P=0.014 ; n=$ 5-6 mice per treatment arm; Figure 3C and Supplemental Figure 3C).

Flow cytometry of paired $\mathrm{OHSV}^{\mathrm{ULBP}}{ }^{-}$-treated and contralateral untreated tumor areas (XFM-Luc:PDGF,Cre) determined a bilateral greater than 2-fold increase of the $\mathrm{CD} 45^{+}$bone marrow-derived immune cell fraction 7 days after virotherapy (Figure 3D). Using a customized nCounter high-sensitivity gene expression array, we detected that viral RNA was locally restricted to treated tumors (Supplemental Figure 3D), thus precluding that viral spread caused the abscopal accumulation of $\mathrm{CD} 45^{+}$cells. 
A

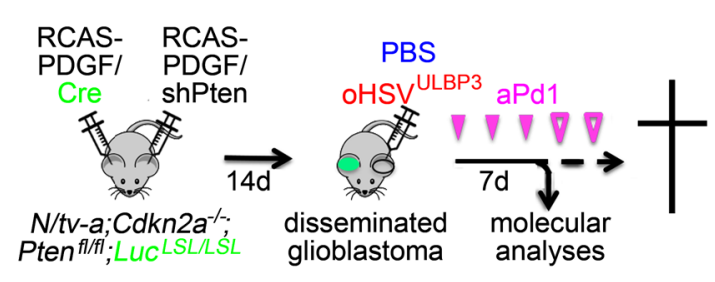

B

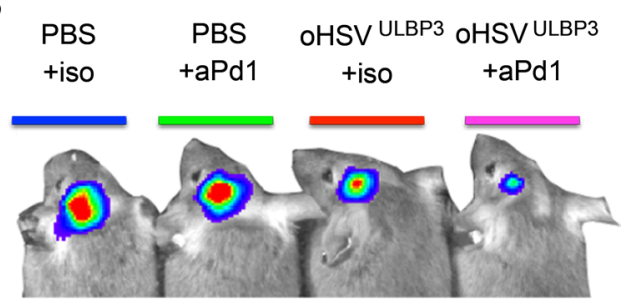

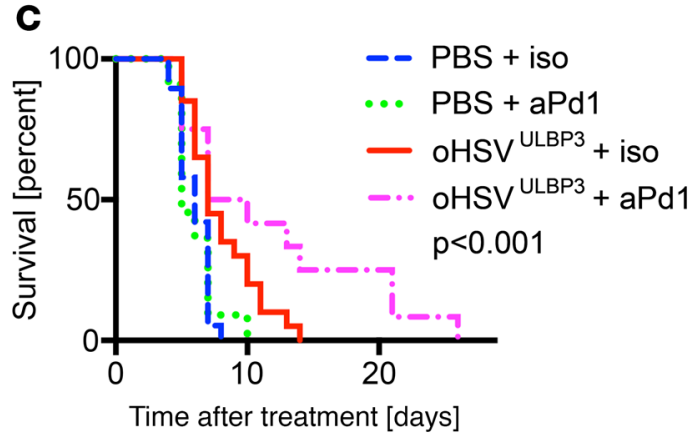

D

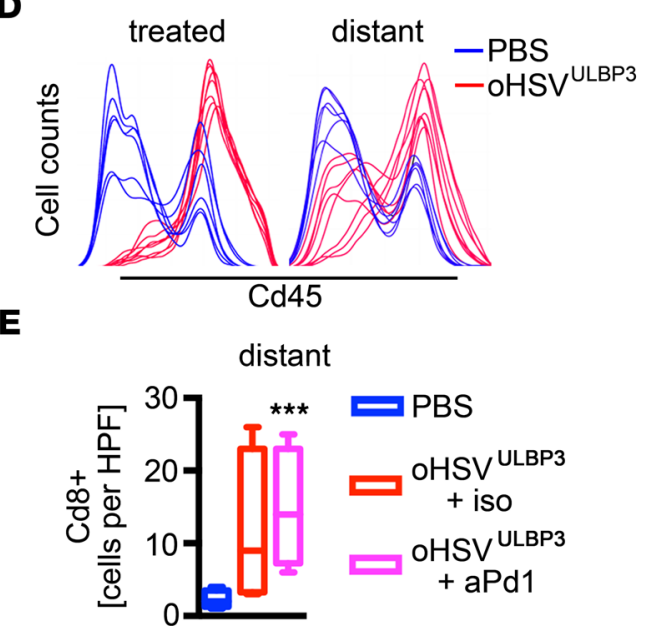

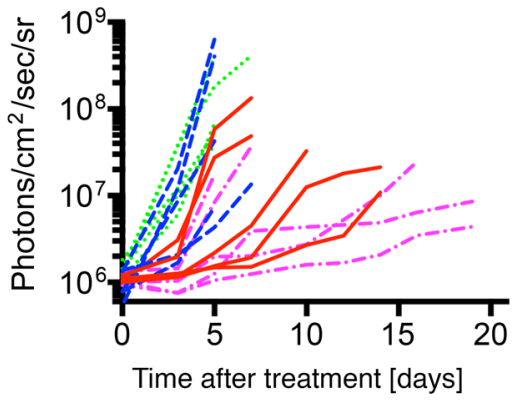

$\mathbf{F}$

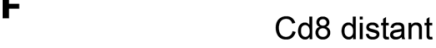

Figure 3. Localized oHSV ${ }^{\mathrm{LBP3}}$ infection elicits a distant immune response and sensitizes distant tumor lesions to anti-PD-1. (A) Experimental setup. (B) Bioluminescence imaging of contralateral, not virally treated tumors in indicated treatment groups. $n=5-6$ mice/group. (C) Symptom-free survival. PBS cotreated with isotype control $(n=19)$ or anti-PD-1 ( $n=11)$; oHSVULBP3 cotreated with isotype control $(n=20)$ or anti-PD-1 ( $n=12)$. Kaplan-Meier curves were compared using the log-rank test. sr, siradian. (D) Flow cytometry-normalized histograms depicting the CD45+ immune cell population in mouse glioblastoma-bearing hemispheres 7 days after injection with PBS or oHSV ${ }^{\text {ULBP3 }}$ (left) and distant, untreated hemispheres from the same mice (right). (E) CD8 ${ }^{+}$cell counts in distant hemispheres upon unilateral treatment with PBS or oHSVULBP3 in combination with anti-PD-1 or isotype 10 mg/kg i.v. every other day. Immunohistochemistry was done on day 7 after initiation of treatment. $n=4$ mice per group. Unpaired, 2 -tailed $t$ test. ${ }^{* *} P<0.001$. The box plots depict the minimum and maximum values (whiskers), the upper and lower quartiles, and the median. The length of the box represents the interquartile range. (F) Representative immunohistochemistry staining of CD8 ${ }^{+}$cells in untreated, tumor-bearing hemispheres. Upper: PBS; lower: oHSVULBP3 cotreated with anti-PD-1. Scale bars: $100 \mu$ m.

There was abscopal memory $\mathrm{T}$ cell formation, activation, and exhaustion. By day 3 after unilateral ${ }_{\text {oHSV }}{ }^{\text {ULPP3 }}$ treatment, there was a bilateral induction of $\mathrm{CD} 44^{+} \mathrm{CD} 62 \mathrm{~L}^{+}$central memory $\mathrm{T}$ cells in both the $\mathrm{CD}^{+}$and $\mathrm{CD}^{+}$tumor-infiltrating lymphocyte fractions (Supplemental Figure 3E), suggesting that the abscopal effect involves $\mathrm{T}$ cell memory. The fraction of $\mathrm{CD}^{+}$tumor-infiltrating lymphocytes positive for the activation markers CD69 or Nkg2d increased simultaneously in virus-treated tumors and abscopally in 5 of 5 mice (Supplemental Figure $3 \mathrm{~F}$ ). In contrast, the $\mathrm{CD}^{+} \mathrm{CD} 44^{-} \mathrm{CD} 62 \mathrm{~L}^{+}$naive $1 \mathrm{ym}$ phocyte fraction was almost completely suppressed bilaterally (Supplemental Figure 3G). The rationale for combining oHSV ${ }^{\mathrm{ULBP} 3}$ with anti-PD-1 was further supported by a 3-fold increase in the fraction of $\mathrm{Pd}^{+}{ }^{+} \mathrm{Tim}^{+}{ }^{+}$exhausted $\mathrm{CD}^{+} \mathrm{T}$ cells in oHSV ${ }^{\mathrm{ULBP}}{ }^{-}$-treated tumors ( 8 of 8 analyzed tumors, $\left.P<0.001\right)$ and a greater than 1.5-fold abscopal increase ( 6 of $8, P=0.022$; Supplemental Figure $3 \mathrm{H}$ ). 
Immunohistochemistry of untreated contralateral tumors also revealed an abscopal increase in total infiltrating $\mathrm{CD}^{+} \mathrm{T}$ cells upon ipsilateral oHSV ${ }^{\mathrm{ULBP} 3}$ treatment in 2 of 4 analyzed tumors $(P=0.13)$, and this trend was further augmented to a mean 12-fold increase in abscopal CD8 ${ }^{+}$tumor infiltration upon cotreatment with anti-PD-1 ( $P=0.003$; Figure 3E). In line with flow cytometry findings, the morphologic notion of an enlarged cytoplasm and vesicles accumulating at the cellular membrane were suggestive of an activated state of these $\mathrm{CD}^{+}$cells (Figure $3 \mathrm{~F}$ ). In summary, these data indicate that oHSV ULBP3 initiates an abscopal $\mathrm{T}$ cell response that can be augmented using immune checkpoint inhibition with anti-PD-1.

Abscopal TAM accumulation and activation upon localized oHSVULBP3 infection. Because the model used here does not contain known nonsynonymous mutations, it remains elusive which (neo-)antigens could have triggered bilateral accumulation and activation of $\mathrm{T}$ cells upon unilateral oHSV ${ }^{\mathrm{ULBP} 3}$ treatment. Human glioblastoma likewise carries only few nonsynonymous mutations (18), but naturally occurring $\mathrm{T}$ cell responses directed against tumor-associated antigens of development-associated genes have been reported (19). Whether similar antigens elicit $\mathrm{T}$ cell responses in mouse glioblastomas is not known. In fact, the observed bilateral influx of activated $\mathrm{T}$ cells could have been an epiphenomenon of a unilateral $\mathrm{T}$ cell response against HSV antigens or against ULBP3, which is a foreign antigen in the mouse too, followed by surveillance of the entire brain. This possibility indicates that direct killing of tumor cells by macrophages could account for the majority of the observed antitumor effect of $\mathrm{oHSV}^{\mathrm{ULBP} 3}$, at least in untreated tumor lesions where viral replication did not occur and where, therefore, foreign antigens were not present.

To explore this possibility, we sought to characterize the $\mathrm{OHSV}^{\mathrm{ULB} 3}$-driven myeloid immune cell infiltrate in more detail. We did time course analyses of tumor-infiltrating $\mathrm{CD}^{4} 5^{+}$cells by flow cytometry of oHSVULBP3-treated tumors and contralateral untreated tumors in the bilateral XFM-Luc:PDGF,Cre glioblastoma model. There was an early bilateral influx of monocytes $\left(\mathrm{CD} 45^{+} \mathrm{CD} 11 \mathrm{~b}^{+} \mathrm{Ly} 6 \mathrm{c}^{+}\right)$in 8 of 8 mice peaking at day 3 after virotherapy in both, ipsilateral treated tumors ( $>5$-fold, $P<0.001$ ), and abscopally ( $>4$-fold, $P<0.001$; Figure 4A). Immunohistochemistry for Iba1 confirmed an abscopal increase of TAM (1.5-fold, $P=0.014)$ in 4 of 4 analyzed tumors upon unilateral oHSV ${ }^{\text {ULBP3 }}$ treatment, and anti-PD-1 treatment further augmented that abscopal effect in 2 of 4 untreated tumors $(P=0.040)$, although we noted no effect of anti-PD-1 monotherapy on TAM accumulation $(P=0.90$; Supplemental Figure $4 \mathrm{~A})$. Interestingly, immunohistochemistry also revealed that ipsilateral treatment with oHSV ULBP3 yielded abscopal relative exclusion of Iba1 ${ }^{+}$TAMs from pseudopalisades and transformation toward a phenotype suggestive of phagocytosis and potentially proinflammatory activation (Figure $4 \mathrm{~B})$. In line with the notion of bilateral macrophage activation upon unilateral treatment with oHSV ${ }^{\text {UBP3 }}$, flow cytometry determined there was a greater than 3 -fold bilateral increase in cells expressing the macrophage activity marker CD44, which persisted by day 14 after unilateral oHSV ${ }^{\mathrm{ULBP} 3}$ infection (Supplemental Figure 4B). There was also upregulation of MHC-II molecules on the cell surface of $\mathrm{CD} 11 \mathrm{c}^{+} \mathrm{MHC}-\mathrm{II}^{+}$cells upon oHSV UBLP3 treatment both ipsilaterally $(P=0.007)$ in 7 of 7 treated tumors and in 6 of 7 contralateral untreated tumors $(P$ $=0.011$; Supplemental Figure $4 \mathrm{C}$ ). Gene expression analyses of tumors contralateral to oHSVuLBP3 treatment were also suggestive of proinflammatory TAM repolarization, including a greater than 4-fold increase of the CD68/CD163 ratio $(P=0.005$; Supplemental Figure 4D), and upregulation of MAPK signaling $(P=0.003)$ and NF- $\mathrm{kB}$ signaling $(P=0.003$; Supplemental Figure 4E).

TAM activation yields upregulation of the immune checkpoint receptor PD-1, and activation of PD-1 by its main ligand, PD-L1, drives immunosuppressive repolarization of TAMs (20). Flow cytometry of bilateral XFM-Luc:PDGF,Cre glioblastomas determined PD-1's presence on more than 70\% of TAMs in both $\mathrm{oHSV}^{\mathrm{ULBP}}$-treated tumors and abscopally, compared with approximately $20 \% \mathrm{PD}-1^{+} \mathrm{TAMs}$ in PBS-treated tumors (Figure 4C). As an add-on to virotherapy, anti-PD-1 treatment was also associated with enrichment of the antigen presentation gene set in both oHSV ${ }^{\mathrm{ULBP3}}$-treated tumor lesions $(P=0.037)$ and abscopally in untreated tumor areas $(P=0.017$; Figure $4, \mathrm{D}$ and $\mathrm{E})$.

Upon treatment with anti-PD-1 alone (10 mg/kg i.v. every other day), nCounter gene expression analysis (5 mice/group) of a myeloid innate immunity panel determined there was enrichment of GO terms associated with proinflammatory processes, including antigen presentation $(P<0.001)$, interferon signaling $(P<0.001)$, and TLR signaling $(P=0.012$; Supplemental Figure $4 \mathrm{~F})$. Similar enrichment patterns could be determined by KEGG gene set enrichment analysis (Supplemental Figure 4G).

Similar analyses applying the nCounter immunology gene panel identified abscopal enrichment of the KEGG gene sets phagosome $(P=0.008)$ and TLR signaling $(P=0.032)$ when anti-PD-1 was added to ${ }_{\text {oHSV }}{ }^{\text {UBP3 }}$. These data indicate that combination with anti-PD-1 augments TAM-driven local and abscopal effects of oHSV $\mathrm{ULBP}^{\mathrm{B}}$. 
A treated

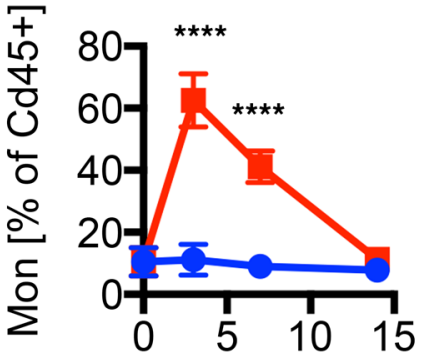

Time post treatment [days]

C
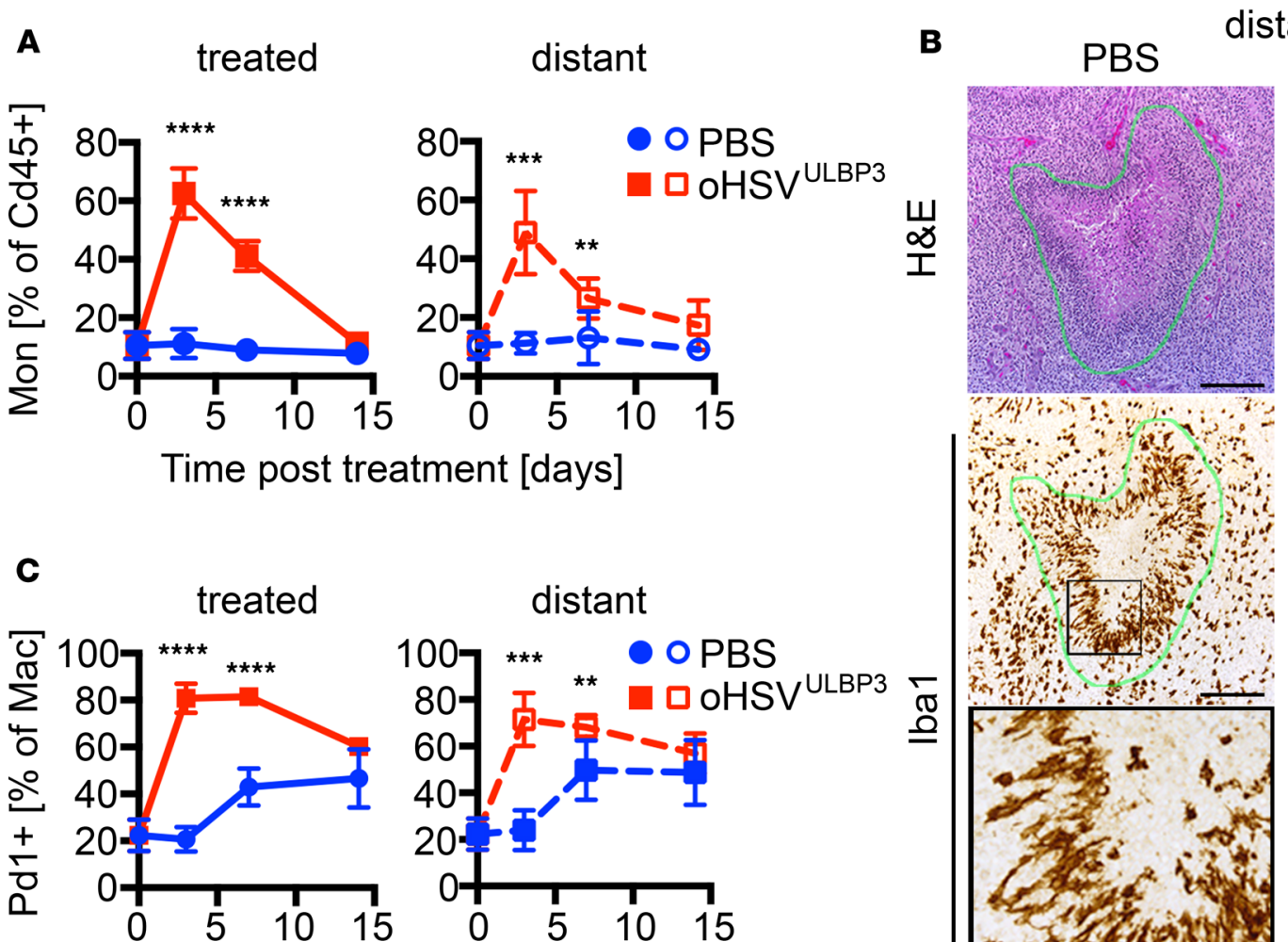

distant

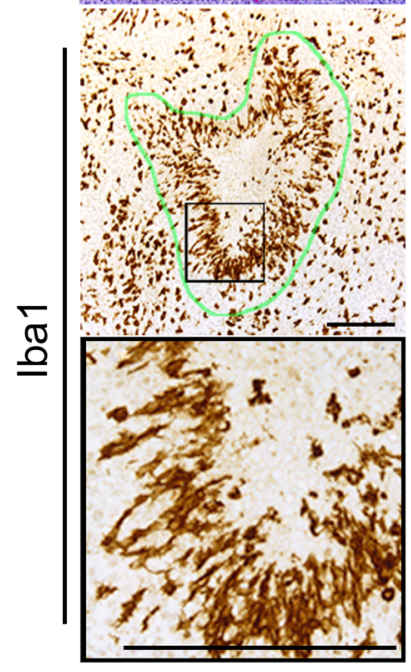

oHSV ULBP3

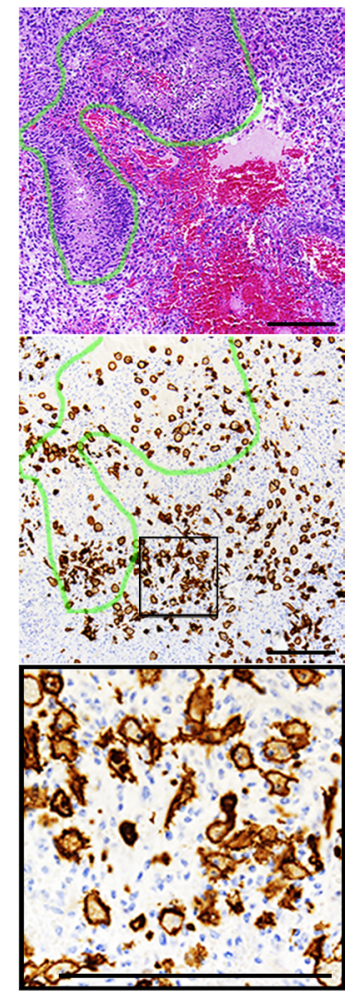

D

up-regulated genes (distant)

$\mathbf{E}$
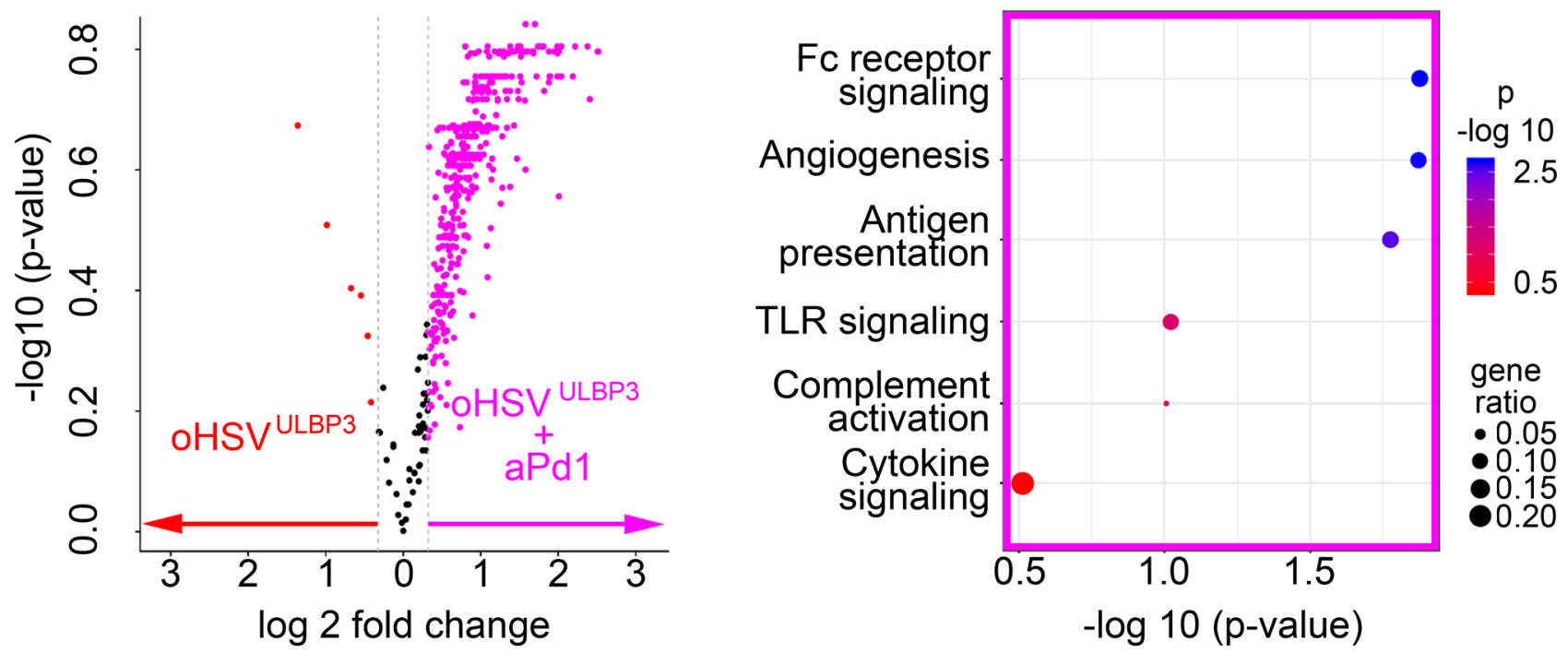

Figure 4. Anti-PD-1 augments oHSVULBP3-driven distant TAM activation. Bilateral XFM-Luc:PDCF,Cre glioblastoma-bearing mice were treated unilaterally as indicated in Figure 3A. (A) Flow cytometry time course analysis of the tumor-infiltrating monocyte fraction (CD45+CD11 b $\left.\mathrm{L}^{+} \mathrm{Ly} 6 \mathrm{c}^{\mathrm{hi}} \mathrm{Ly} 6 \mathrm{~g}^{-}\right) . n=5-8 \mathrm{mice}^{-}$ group/time point; mean \pm SEM; unpaired, 2 -tailed $t$ test. ${ }^{*} P<0.01 ;{ }^{* *} P<0.001$; and ${ }^{* * *} P<0.0001$. (B) Representative H\&E or Iba1 immunohistochemistry stains in adjacent slides of untreated hemispheres (distant), contralateral to tumors injected with PBS or oHSVuLBP3. Green lines, pseudopalisading

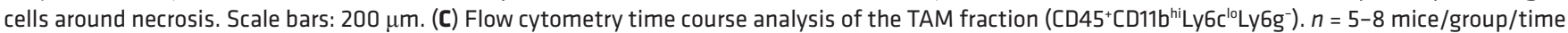
point; mean \pm SEM; unpaired, 2-tailed $t$ test. ${ }^{* *} P<0.01$; ${ }^{* *} P<0.001 ;{ }^{* * *} P<0.0001$. (D) Volcano plot of differentially expressed myeloid genes in distant tumors, contralateral to oHSV ULBP3 treatment, with versus without cotreatment with anti-PD-1 ( $n=5$ per group). (E) Gene set enrichment analysis of genes upregulated in distant tumors upon addition of anti-PD-1 to oHSVuLBP3 (magenta in D). 


\section{Discussion}

Oncolytic virotherapy has emerged as a viable approach to revert immunosuppression in immunologically "cold" cancers, including glioblastoma (2, 5-7, 21-29). Here, we have used an miR-124-based oHSV attenuation system that circumvents interferon sensitivity conveyed by the commonly used infected cell protein 34.5 loss-of-function attenuation strategy (12). Arming our miR-124-attenuated oHSV with ULBP3 promoted a localized and abscopal anticancer immune response that was sensitized to anti-PD-1 in a clinically relevant genetic glioblastoma model (4).

The notion of direct tumor cell killing by activated macrophages is consistent with preclinical reports in other entities, e.g., nonhypermutated colon cancer (30). Moreover, the proinflammatory effect of antiPD-1 on myeloid cells in virus-injected and distant, untreated tumor areas underscores the role of PD-1 signaling as an inhibitory pathway in tumor macrophages (20) and supports combination treatments of oHSV with anti-PD-1 irrespective of tumor T cell infiltration.

Extensive TAM infiltration and activation followed by an adaptive immune response following virotherapy has been reported previously, including in syngeneic glioblastoma models $(27,31)$. Beyond the necessity of macrophage repolarization for establishing an adaptive immune response, our data also support previous reports on the role of TAMs for the clearance of oHSV-infected cells $(32,33)$. Similar effects of NK cells have also been reported to limit the efficacy of oHSV treatment (34); albeit in our model, the downregulation of activation markers and the low abundance of NK cells suggest that mainly TAMs have limited viral spreading.

A previous report on the combination treatment of an oHSV with immune checkpoint inhibition in a therapeutically challenging, immune-suppressed glioblastoma model demonstrated that TAMs and T cells are both required to exert efficacious antitumor effects (27). The observed dominance of a myeloid immune response in our model may have several causes: (a) gene expression changes in the lymphocytic compartment were likely covered by myeloid- and tumor-associated gene expression patterns because of the relatively lower abundance of lymphocytes; (b) our genetic in situ modeling approach does not convey potentially immunogenic neoantigens, thus likely limiting the efficacy of $\mathrm{T}$ cell infiltration; and (c) the short survival time of our model may not suffice to fully establish an anticancer $\mathrm{T}$ cell response, i.e., the observed infiltration of activated $\mathrm{T}$ cells may be no more than the onset of such a response. Therefore, depleting macrophages (e.g., by using clodronate liposomes) will likely have a more prominent effect than depleting $\mathrm{CD} 8^{+} \mathrm{T}$ cells in our model, but the translational relevance of such findings will be limited by the above-mentioned characteristics of our model.

Yet, our model also conveys major advantages over commonly used syngeneic glioblastoma models, including (a) the omission of cell culture artifacts by inducing tumors in situ from each mouse's own cells, (b) the presence of oncogenic driver events that are indeed present in human glioblastoma (versus random oncogenic transformation by chemical induction or ex vivo overexpression of oncogenes that are rare in glioblastoma, e.g., mutated RAS), and (c) a distinctive lymphocyte depletion in untreated tumors.

In summary, the data presented herein support further development of virotherapy as a means to sensitize cancers to immune checkpoint inhibition, including in diffusely infiltrating brain cancers where only a subset of cells can be infected.

\section{Methods}

Genetically engineered mouse glioblastoma model. The RCAS/tv-a model system was used to generate mouse glioblastomas (35). N/tva Cdkn2a/b-1- Pten ${ }^{\text {fl/fl}}$ LSL Luciferase mice (36) were used as genetic backgrounds for RCAS-mediated glioblastoma formation. For a bilateral tumor model, following introduction of RCAS-PDGFB with $R C A S$-Cre into 1 hemisphere and RCAS-PDGFB with RCAS-shPten into the contralateral hemisphere for bioluminescence, 2 genetically similar tumors are present where 1 tumor lacks luciferase expression (PDGFB/ shPten; treated glioblastoma) and another tumor has luciferase expression (PDGFB/Pten ${ }^{-/}$Luciferase; distant glioblastoma for assessment of abscopal response). Tumors were generated in 4- to 6-week-old male and female mice injected with $2 \times 10^{5} \mathrm{DF} 1$ cells delivered with a 30-gauge needle attached to a Hamilton syringe and stereotactic fixation device (Stoelting). Cells were injected into the frontal cortex of each hemisphere: coordinates were lateral to the bregma $3 \mathrm{~mm}$ at a depth of $1 \mathrm{~mm}$. Treatment began 14 days after tumor formation.

The RCAS vectors used were generated as previously described (4). Briefly, DF1 cells were purchased from ATCC and grown at $39^{\circ} \mathrm{C}$. Transfections with RCAS vectors were performed using the Fugene 6 transfection kit (Roche E2691). Nestin-specific, Cre-recombinase-responsive, PDGF luciferase-expressing mouse models were monitored until they developed signs of tumor burden, including lethargy, poor grooming, weight loss, macrocephaly, seizure, and paralysis. 
Virotherapy and drug treatments in vivo. Adult mice were sedated with isoflurane. The same coordinates as for tumor initiation were used for oncolytic virus treatment. Injection of $10^{6} \mathrm{PFU}$ of indicated oHSV strains in $1 \mu \mathrm{L}$ PBS, or $1 \mu \mathrm{L}$ PBS alone, took place over 2 minutes using a 30-gauge needle attached to a Hamilton syringe and stereotactic fixation device. In the double-tumor model, only 1 tumor was injected. The same stereotactic coordinates as for tumor formation were used.

Endotoxin-low $(\leq 0.001 \mathrm{EU} / \mu \mathrm{g} ; \leq 1 \mathrm{EU} / \mathrm{mg})$ anti-CTLA4, anti-PD-L1, anti-PD-1, or indicated isotype controls ( $10 \mu \mathrm{g} / \mathrm{g}$ mouse body weight, see Supplemental Table 1) were administered starting on the day of virotherapy and then every other day until endpoint. Bioluminescence signals were obtained for each treatment group every 2 to 3 days following treatment. Animals were sacrificed upon development of neurologic symptoms as defined by the Institutional Animal Care and Use Committee.

Bioluminescence. Bioluminescence imaging was obtained on the Xenogen IVIS 100 system. Mice with tumors expressing luciferase were anesthetized with isoflurane before retro-orbital injection with $75 \mathrm{mg} /$ $\mathrm{kg}$ body weight D-luciferin (Caliper Life Sciences, 119222). Promptly after injection, repeat images were acquired for 3 to 5 minutes to ensure that the plateau phase of photon emission was reached. Total flux (photon seconds ${ }^{-1}$ ) was obtained from each mouse.

Immunohistochemistry and immunofluorescence. Mouse brains were fixed in formalin and embedded in paraffin, then sectioned at $5 \mu \mathrm{m}$ and stained by routine H\&E, as well as immunohistochemistry (IHC) or immunofluorescence (IF). Automated IHC and antigen retrieval for IF were performed on the Discovery XT (Ventana Medical Systems Inc.) according the manufacturer's standard protocol. For IF, after washing with TBS, a blocking step with TBS containing 10\% donkey serum of 1 hour, sections were rinsed with TBS and incubated in a mixture of indicated antibodies of each series in TBS containing $2 \%$ BSA overnight at $4^{\circ} \mathrm{C}$. After washing in TBS, a cocktail of indicated secondary antibodies (Supplemental Table 1) was applied for 4 hours at room temperature. Sections were washed again, mounted, and coverslipped in polyvinyl alcohol with diazabicyclo-octane (DABCO) as an antifading agent. Images were acquired using a Zeiss LSM 780 NLO confocal microscope.

Gene expression analysis. Paraffin-embedded tissue sections were macrodissected, and RNA was extracted using the RNeasy FFPE kit (Qiagen, 73504). Contaminating DNA was removed with DNAse (Thermo Fisher Scientific, EN0525). We hybridized $50 \mathrm{ng}$ of total RNA at $96^{\circ} \mathrm{C}$ overnight with the nCounter mouse immunology and myeloid innate immunity panels (NanoString; XT-CSO-MIM1-12, XT-CSO-MMII2-12). Hybridized samples were analyzed for frequency of each RNA species using the nCounter SPRINT profiler (NanoString). Raw mRNA abundance frequencies were analyzed using the nSolver analysis software 2.5 pack. Normalization factors were derived from the geometric mean of housekeeping genes, after quality control with built-in negative and positive controls.

Flow cytometry. Animals were sacrificed, and their brains were removed from skulls immediately after cardiac perfusion with $10 \mathrm{~mL}$ of ice-cold PBS. Mouse brains were mechanically dissociated and gently forced through a $70-\mu \mathrm{m}$ strainer followed by rinsing with $50 \mathrm{~mL}$ of Hanks' balanced salt solution (HBSS) plus $10 \%$ FBS. Cells were pelleted by centrifugation ( $500 \mathrm{~g}$ for $10 \mathrm{~min}$ at $4^{\circ} \mathrm{C}$ ) and suspended in red blood cell (RBC) lysis buffer (MilliporeSigma) ( $5 \mathrm{~mL} /$ brain, 1 minute on ice). RBC lysis was quenched by adding 3 volumes of HBSS plus $10 \%$ FBS, and cells were centrifuged at $500 \mathrm{~g}$ for 10 minutes at $4^{\circ} \mathrm{C}$. To remove myelin, cells were suspended in $10 \mathrm{~mL}$ of $30 \%$ Percoll (GE Healthcare) and centrifuged at $700 \mathrm{~g}$ for 10 minutes at room temperature without break. The myelin layer at the top was removed, and the cell pellet was resuspended in HBSS plus $10 \%$ FBS, run through a $40-\mu \mathrm{m}$ cell strainer, and centrifuged at $500 \mathrm{~g}$ for 10 minutes at $4^{\circ} \mathrm{C}$. The cell pellet was resuspended in cold PBS plus $2 \%$ FBS to perform cell count and FACS staining. Used antibodies are listed in Supplemental Table 1. Samples were analyzed on an LSR II flow cytometer with FACSDiva software (BD Biosciences). Compensation and analysis were performed with FlowJo software (Tree Star), and gates were defined by fluorescence minus 1 controls.

ULBP3-binding studies. Human peripheral blood mononuclear cells were isolated from whole blood via density gradient over Ficoll-Paque 1.077 (Fisher Scientific, Thermo Fisher Scientific, 45-001-751). The buffy coat layer was isolated, and cells were washed in $1 \times$ PBS twice to remove platelets $(300 \mathrm{~g})$. Cells were used fresh or frozen at $10 \times 10^{6} / \mathrm{mL}$ to $20 \times 10^{6} / \mathrm{mL}$ in freeze medium (90\% FBS, $\left.10 \% \mathrm{DMSO}\right)$ for later use. Mouse spleens were harvested from naive C57BL/6 mice via mechanical dissociation and filtration over a 70- $\mu \mathrm{m}$ filter. Erythrocytes were removed using ACK lysing buffer (Fisher Scientific, Thermo Fisher Scientific, A1049201). Cells were used fresh or frozen at 10-20 $\times 10^{6} / \mathrm{mL}$ in freeze medium (90\% FBS, $10 \%$ DMSO) for later use. Staining was carried out in 96-well, round-bottom plates with $1 \times 10^{6}$ cells/well. Cells 
were washed and stained in Stain Buffer (BD Biosciences, 554657) with 2 mM EDTA. Live/dead staining was performed using Live/Dead Fixable Yellow Amine Stain (Life Technologies; L34968). Fc receptors were blocked to prevent nonspecific binding of antibodies or rhULBP3-Fc chimera protein (R\&D Systems; 1517-UL-50) using TruStain FcX (BioLegend; mouse: 101320; human: 422302) and following manufacturer's protocol. Cells were then treated with or without 10 or $20 \mu \mathrm{g} / \mathrm{mL}$ anti-NKG2D blocking antibody (mouse or human, respectively) (R\&D Systems; human: MAB139; mouse: MAB1547) for 20 minutes at room temperature. Cells were washed, then incubated with species-appropriate flow panels (below) and 5 or $20 \mu \mathrm{g} / \mathrm{mL}$ rhULBP3-Fc (mouse or human, respectively) for 20 minutes at room temperature. Cells were washed and stained with APC anti-human IgG-Fc secondary antibody (R\&D Systems; FAB110A). Finally, cells were washed and resuspended in FluorFix fixation buffer (BioLegend, 422101). Samples were run on a BD Biosciences LSR Fortessa; data were acquired using FACSDiva software (BD Biosciences) and analyzed using FlowJo software (Tree Star).

In vitro cytotoxicity assay. Early-passage, XFM-Luc-derived primary mouse glioblastoma cells were main-

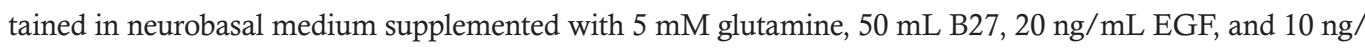
mL FGF. Cells were separated using accutase (Stemcell Technologies) and were seeded at a density of 5000 per well in $200 \mu \mathrm{L}$ medium in 96-well plates overnight. Subsequently, cells were treated with oHSV at indicated $\log _{10}$ increasing doses in quadruplicate for 48 hours. Cell-free medium was used as a negative control, and cells lysed with $1 \%$ Triton X-100 were the positive control. The microplate was then centrifuged at $250 \mathrm{~g}$ for 10 minutes and $100 \mu \mathrm{L}$ medium transferred into a corresponding flat-bottom, 96-well plate with $100 \mu \mathrm{L}$ freshly prepared reaction mixture added to each well. After incubation for 30 minutes at room temperature protected from light, the absorbance at $490 \mathrm{~nm}$ was measured using a standard plate reader.

Bioinformatics. RCC files were analyzed with nSolver software. A statistical cutoff of $\log _{2}$-fold change greater than 1.25 was used to find differentially regulated genes. GO and KEGG enrichment analyses were done using R Bioconductor packages cluster Profiler v 3.4.4 (37), and dot plots were made using R Bioconductor package DOSE (38) and ggplot2 (39). For both GO and KEGG analysis, the gene universe was restricted to the genes arrayed in both the panels.

Statistics. Statistical analysis was performed using GraphPad Prism 7. Unpaired, 2-tailed $t$ test was used unless otherwise indicated. For survival studies, Kaplan-Meier curves were compared using the log-rank test. Linear regression or 2-way ANOVA was done to compare groups in dose-response and time course analyses. $P$ values below 0.05 were considered statistically significant.

Study approval. Animal studies were carried out in accordance with the Guide for the Care and Use of Laboratory Animals, as adopted by the NIH (National Academies Press, 2011). Animal research was prospectively approved by the Institutional Animal Care and Use Committee of the Fred Hutchinson Cancer Research Center (animal assurance institutional A3226-01; protocol 50842). Informed consent was obtained from donors of peripheral blood mononuclear cells.

\section{Author contributions}

$\mathrm{ECH}$ and HGW designed the study and interpreted the data. $\mathrm{AMH}$ and $\mathrm{HZ}$ designed the flow cytometry panel. ECH, HGW, SA, and HZ analyzed the data. HGW and SA created the figures. HGW, HZ, MB, and JSC collected data. ECH and HGW drafted the manuscript. HGW, HZ, FS, SA, PG, PJC, NA, JSC, LM, SSP, CE, AH, MB, JY, JZ, DK, RHP, MW, MF, CQ, JCG, AMH, and ECH contributed to the writing and revision of the manuscript and approved the final version.

\section{Acknowledgments}

The authors would like to thank Edward M. Kennedy and Terry Farkaly for designing the customized nCounter panel to detect viral genes. This research was supported by P2SKP3_158656 from the Swiss National Science Foundation (to HGW), CA60882-01A1 (to ECH), R01 CA195718-01 (to ECH), U54 CA193461-01 (to ECH), R01 CA175052 (to JCG), R01 CA222804 (to JCG), and P01 CA163205 (to EA Chiocca, project 1: JCG) from the NIH and by a grant from Oncorus (to ECH).

Address correspondence to: Eric C. Holland, Fred Hutchinson Cancer Research Center, PO Box 19024, C3-168, Seattle, Washington 98109-1024, USA. Phone: 206.667.6117; Email: eholland@fredhutch.org. 
1. Ostrom QT, Gittleman H, Truitt G, Boscia A, Kruchko C, Barnholtz-Sloan JS. CBTRUS Statistical Report: primary brain and other central nervous system tumors diagnosed in the United States in 2011-2015. Neuro Oncol. 2018;20(supp1_4):iv1-iv86.

2. Lim M, Xia Y, Bettegowda C, Weller M. Current state of immunotherapy for glioblastoma. Nat Rev Clin Oncol. 2018;15(7):422-442.

3. Thorsson V, et al. The immune landscape of cancer. Immunity. 2018;48(4):812-830.e14.

4. Ozawa T, et al. Most human non-GCIMP glioblastoma subtypes evolve from a common proneural-like precursor glioma. Cancer Cell. 2014;26(2):288-300.

5. Lang FF, et al. Phase I study of DNX-2401 (Delta-24-RGD) oncolytic adenovirus: replication and immunotherapeutic effects in recurrent malignant glioma. J Clin Oncol. 2018;36(14):1419-1427.

6. Desjardins A, et al. Recurrent glioblastoma treated with recombinant poliovirus. N Engl J Med. 2018;379(2):150-161.

7. Samson A, et al. Intravenous delivery of oncolytic reovirus to brain tumor patients immunologically primes for subsequent checkpoint blockade. Sci Transl Med. 2018;10(422):eaam7577.

8. Reifenberger G, Wirsching HG, Knobbe-Thomsen CB, Weller M. Advances in the molecular genetics of gliomas - implications for classification and therapy. Nat Rev Clin Oncol. 2017;14(7):434-452.

9. Omuro A, et al. Nivolumab with or without ipilimumab in patients with recurrent glioblastoma: results from exploratory phase I cohorts of CheckMate 143. Neuro Oncol. 2018;20(5):674-686.

10. Johanns TM, et al. Endogenous neoantigen-specific CD8 T cells identified in two glioblastoma models using a cancer immunogenomics approach. Cancer Immunol Res. 2016;4(12):1007-1015.

11. Genoud V, et al. Responsiveness to anti-PD-1 and anti-CTLA-4 immune checkpoint blockade in SB28 and GL261 mouse glioma models. Oncoimmunology. 2018;7(12):e1501137.

12. Mazzacurati L, et al. Use of miRNA response sequences to block off-target replication and increase the safety of an unattenuated, glioblastoma-targeted oncolytic HSV. Mol Ther. 2015;23(1):99-107.

13. Sutherland CL, Rabinovich B, Chalupny NJ, Brawand P, Miller R, Cosman D. ULBPs, human ligands of the NKG2D receptor, stimulate tumor immunity with enhancement by IL-15. Blood. 2006;108(4):1313-1319.

14. Bauer S, et al. Activation of NK cells and T cells by NKG2D, a receptor for stress-inducible MICA. Science. 1999;285(5428):727-729.

15. Haas J, et al. The Protein Model Portal - a comprehensive resource for protein structure and model information. Database (Oxford). 2013;2013:bat031.

16. Huttlin EL, et al. Architecture of the human interactome defines protein communities and disease networks. Nature. 2017;545(7655):505-509.

17. Jinushi M, Hodi FS, Dranoff G. Therapy-induced antibodies to MHC class I chain-related protein A antagonize immune suppression and stimulate antitumor cytotoxicity. Proc Natl Acad Sci U S A. 2006;103(24):9190-9195.

18. Brennan CW, et al. The somatic genomic landscape of glioblastoma. Cell. 2013;155(2):462-477.

19. Neidert MC, et al. The natural HLA ligandome of glioblastoma stem-like cells: antigen discovery for T cell-based immunotherapy. Acta Neuropathol. 2018;135(6):923-938.

20. Gordon SR, et al. PD-1 expression by tumour-associated macrophages inhibits phagocytosis and tumour immunity. Nature. 2017;545(7655):495-499.

21. Lawler SE, Speranza MC, Cho CF, Chiocca EA. Oncolytic viruses in cancer treatment: a review. JAMA Oncol. 2017;3(6):841-849.

22. Kaufman HL, Kohlhapp FJ, Zloza A. Oncolytic viruses: a new class of immunotherapy drugs. Nat Rev Drug Discov. 2015;14(9):642-662.

23. Lubin JA, Zhang RR, Kuo JS. Zika virus has oncolytic activity against glioblastoma stem cells. Neurosurgery. 2018;82(5):E113-E114.

24. Brown MC, et al. Cancer immunotherapy with recombinant poliovirus induces IFN-dominant activation of dendritic cells and tumor antigen-specific CTLs. Sci Transl Med. 2017;9(408):eaan4220.

25. Chiocca EA, et al. A phase I open-label, dose-escalation, multi-institutional trial of injection with an E1B-Attenuated adenovirus, ONYX-015, into the peritumoral region of recurrent malignant gliomas, in the adjuvant setting. Mol Ther. 2004;10(5):958-966.

26. Hogan DJ, et al. Molecular analyses support the safety and activity of retroviral replicating vector toca 511 in patients. Clin Cancer Res. 2018;24(19):4680-4693.

27. Saha D, Martuza RL, Rabkin SD. Macrophage polarization contributes to glioblastoma eradication by combination immunovirotherapy and immune checkpoint blockade. Cancer Cell. 2017;32(2):253-267.e5.

28. Markert JM, et al. A phase 1 trial of oncolytic HSV-1, G207, given in combination with radiation for recurrent GBM demonstrates safety and radiographic responses. Mol Ther. 2014;22(5):1048-1055.

29. Harrow S, et al. HSV1716 injection into the brain adjacent to tumour following surgical resection of high-grade glioma: safety data and long-term survival. Gene Ther. 2004;11(22):1648-1658.

30. Halama N, et al. Tumoral immune cell exploitation in colorectal cancer metastases can be targeted effectively by anti-CCR5 therapy in cancer patients. Cancer Cell. 2016;29(4):587-601.

31. Hardcastle J, et al. Immunovirotherapy with measles virus strains in combination with anti-PD-1 antibody blockade enhances antitumor activity in glioblastoma treatment. Neuro Oncol. 2017;19(4):493-502.

32. Meisen WH, et al. The impact of macrophage- and microglia-secreted TNF $\alpha$ on oncolytic HSV-1 therapy in the glioblastoma tumor microenvironment. Clin Cancer Res. 2015;21(14):3274-3285.

33. Delwar ZM, Kuo Y, Wen YH, Rennie PS, Jia W. Oncolytic virotherapy blockade by microglia and macrophages requires STAT1/3. Cancer Res. 2018;78(3):718-730.

34. Alvarez-Breckenridge CA, et al. NK cells impede glioblastoma virotherapy through NKp30 and NKp46 natural cytotoxicity receptors. Nat Med. 2012;18(12):1827-1834.

35. Hambardzumyan D, Amankulor NM, Helmy KY, Becher OJ, Holland EC. Modeling adult gliomas using RCAS/t-va technology. Transl Oncol. 2009;2(2):89-95.

36. Hu X, Pandolfi PP, Li Y, Koutcher JA, Rosenblum M, Holland EC. mTOR promotes survival and astrocytic characteristics induced by Pten/AKT signaling in glioblastoma. Neoplasia. 2005;7(4):356-368.

37. Yu G, Wang LG, Han Y, He QY. clusterProfiler: an R package for comparing biological themes among gene clusters. OMICS. 2012;16(5):284-287. 
38. Yu G, Wang LG, Yan GR, He QY. DOSE: an R/Bioconductor package for disease ontology semantic and enrichment analysis. Bioinformatics. 2015;31(4):608-609.

39. Wickham H. ggplot2: Elegant Graphics for Data Analysis. New York, New York, USA: Springer; 2016. 